\title{
SHOCKS, OUTFLOWS AND BUBBLES: NEW VIEWS ON PULSARS AND THEIR WINDS
}

\author{
BRYAN M. GAENSLER \\ Harvard-Smithsonian Center for Astrophysics, \\ 60 Garden Street $M S-6$, \\ Cambridge MA 02138, USA \\ E-mail: bgaensler@cfa.harvard.edu
}

\begin{abstract}
A typical young pulsar slows down at an imperceptible rate, its spin period increasing by less than 10 microseconds over the course of a year. However, the inertia of a pulsar is so extreme that to effect this tiny change in rotation rate, the star must dissipate about $10^{46}$ ergs of kinetic energy. Observations of pulsars and their surroundings demonstrate that this "spin-down energy" is expelled into the pulsar's surroundings in spectacular fashion, in the form of a relativistic wind of charged particles and magnetic fields. In this review I highlight some recent observational results on pulsar winds at radio, X-ray and optical wavelengths, and explain what we can learn from these data about shock structure, particle acceleration and the interstellar medium.
\end{abstract}

\section{Introduction}

Pulsars are thought to be born with very rapid initial periods, typically 10$100 \mathrm{~ms}$ (see Migliazzo et al. 2002). The rotational energy of a new-born neutron star can thus be very large, potentially approaching the $\sim 10^{51} \mathrm{ergs}$ of kinetic energy released in the surrounding supernova explosion. Observationally, it can be easily established that the spacing between the regular radio pulses seen from these sources is steadily increasing, demonstrating that all young pulsars are slowing down. This "spin-down" implies that a pulsar's enormous reservoir of kinetic energy is being dissipated at a furious rate - for the youngest sources we find $\dot{E} \equiv I \omega \dot{\omega} \approx 10^{35}-10^{39} \mathrm{erg} \mathrm{s}^{-1}$, where $\omega$ is a pulsar's angular frequency of rotation, $I \equiv 10^{45} \mathrm{~g} \mathrm{~cm}^{2}$ is the moment of inertia usually adopted for such sources, and $\dot{E}$ is the pulsar's "spin-down luminosity".

Ultimately this energy dissipation is due to the torques produced by extreme electromagnetic fields on and near the surface of the neutron star; indeed the observed values of $\omega$ and $\dot{\omega}$ are routinely used to infer a neutron 
star's surface magnetic field (Manchester \& Taylor 1977). Further from the star, the bulk of the energy associated with these torques goes into a relativistic $\left(\gamma \approx 10^{6}\right)$ magnetized wind (Michel 1969), through which the star ultimately deposits its rotational energy into its surroundings. (Only a tiny fraction of this spin-down luminosity goes into generating the coherent emission from the magnetic poles which results in the star's characteristic pulsations.)

When a relativistic pulsar wind is confined by external pressure, this source become directly observable as a pulsar wind nebulae $(\mathrm{PWN})^{\mathrm{a}}$, of which the Crab Nebula is the most spectacular example. These sources are our best guide to the processes through which a pulsar loses its energy and couples to its surroundings. Because PWNe are close enough to be spatially resolved, they provide a wonderful laboratory for directly observing the physics of particle acceleration and strong shocks, processes which manifest themselves in a variety of other situations in high-energy astrophysics.

In this review, I summarize what we know about the overall properties and detailed structure of PWNe, with particular emphasis on the flood of recent results which have revitalized this field.

\section{Pulsar Wind Nebulae}

Broadly speaking, there are three types of PWN, reflecting the evolutionary state of the system and the ambient conditions (see Chevalier 1998 for a more detailed discussion).

A very young ( $1000 \mathrm{yr})$ pulsar is typically embedded inside an equally young supernova remnant (SNR). The pulsar's wind is significantly overpressured with respect to the cold ejecta inside the SNR shell. The PWN thus expands rapidly in all directions: one typically observes a quasispherical radio and X-ray nebula, with the pulsar at its center. The Crab Nebula, as shown in Figure 1, is the prototype of such sources (although in this case the surrounding SNR has not yet been directly detected). I and others have in the past referred to these sources as "static PWNe", to differentiate them from bow-shock nebulae as described in $\S 2.3$ below. However, given that these sources are rapidly expanding, this terminology can be confusing — here I prefer to call these sources "bubble" PWNe, to reflect the fact that (deviations from spherical symmetry aside) these sources are simply expanding bubbles.

"sometimes also referred to as a "plerion" 
At later stages in evolution ( $~ 10000 \mathrm{yr}$ ), the SNR evolves towards the "Sedov-Taylor" phase of evolution, in which a reverse shock fills the SNR cavity with shocked ejecta. The collision between this reverse shock and the expanding PWN has a dramatic effect, crushing and compressing the pulsar nebula (e.g. Blondin et al. 2001). The resulting PWN can have drastically different appearances in the radio and X-ray bands, with the pulsar substantially offset from the center of the PWN, and the PWN offset from the center of the SNR.

At much later times, the SNR has dissipated and the pulsar is now moving at a space velocity of typically $\sim 500 \mathrm{~km} \mathrm{~s}^{-1}$ through ambient gas. The ram pressure of the pulsar's wind generates a bow shock, which can be seen as a cometary structure in radio and X-rays, and also possibly in $\mathrm{H} \alpha$ and other optical lines. PSR B1957+20, shown in Figure 2, is a beautiful example of such a system.

\subsection{Bubble Pulsar Wind Nebulae: Overall Properties}

"Bubble" PWNe, as typified by the Crab Nebula, characteristically have a centrally filled morphology at all wavebands and show significant levels of linear polarization. Furthermore, many of these sources are increasingly smaller in spatial extent at progressively higher energies. These observations provide convincing evidence that it is synchrotron emission which we detect from these sources, generated by relativistic electrons and positrons supplied by the central pulsar.

In many of these sources, the synchrotron lifetimes of emitting particles at radio and X-ray energies are significantly more than and less than the age of the source, respectively. This implies that the radio luminosity of such a PWN traces the integrated history of the pulsar's spin-down since birth, while the X-ray luminosity traces the pulsar's current behavior. When combined, these data can be used to build up a broadly consistent picture for the pulsar's evolution and current properties.

The broadband spectrum of such PWNe is inevitably more complicated than a single power law, however, generally showing multiple spectral breaks. Such spectral breaks are often attributed to synchrotron losses, from which the nebular magnetic field can then be inferred (e.g. Frail et al. 1996). However, these spectral features can also be produced by sudden changes in the pulsar's rate of spin-down or by intrinsic breaks in the underlying particle spectrum (Pacini \& Salvati 1973; Woltjer et al. 1997). We currently do not have a good understanding of how to interpret the broad- 
band spectral features of PWNe, and caution should therefore be exercised in interpreting them.

\subsection{Bubble Pulsar Wind Nebulae: Structure}

At the most basic level, a bubble PWNe can be simply approximated as consisting of three zones, as shown in Figure 1. In the inner regions near the pulsar, a relativistic but cold particle wind is launched from the pulsar light cylinder. This wind is not directly observable.
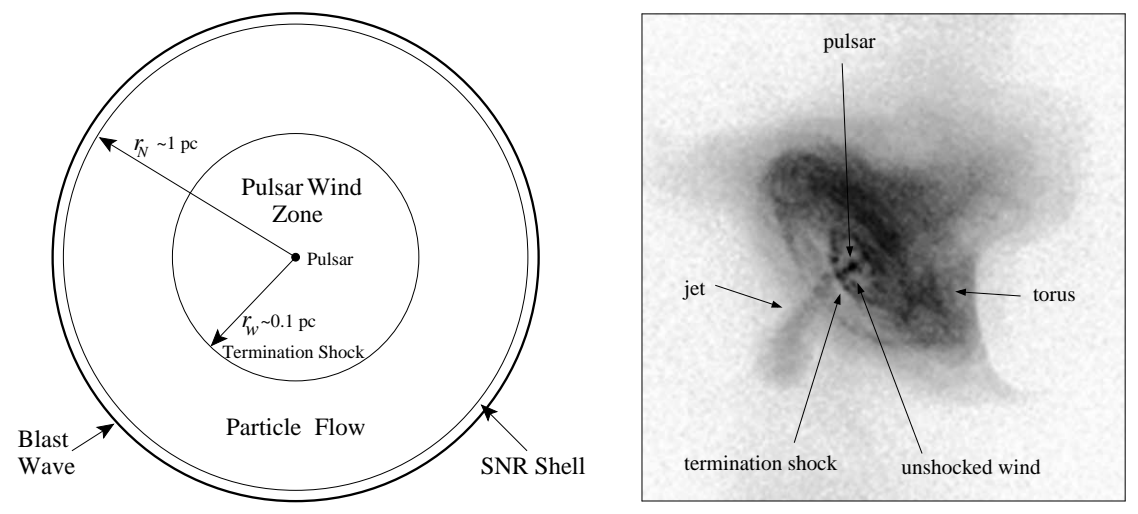

Figure 1. Cartoon of a bubble PWN (left; Slane 2002), compared to a Chandra X-ray image of the Crab Nebula (right; Weisskopf et al. 2002).

At some distance $r_{w}$ from the pulsar, the pressure of the outflowing pulsar wind is balanced by gas and magnetic pressure in the surrounding emitting region. At this termination shock, particles are thermalized and accelerated up to X-ray emitting energies. Downstream of the termination shock, the flow continues to decelerate, and generates observable synchrotron emission.

While there is considerable support for the validity of this simple picture, until recently only the outer, emitting, region of most PWNe had been identified. There have been remarkable advances in this regard with the advent of the Chandra X-ray Observatory, whose unparalleled spatial resolution has allowed us to delineate the termination shock and the region within it in numerous PWNe, as shown in Figure 1. Because the termination shock marks the point at which particles are accelerated and the wind shocks, data on this region are our best handle yet on the process by which the pulsar wind couples with and reacts to its environment. 
Chandra has demonstrated that in many PWNe the termination shock clearly has a toroidal, rather than spherical, geometry, suggesting that the pulsar wind is focused into an equatorial flow, as is indeed expected for an oblique rotator (e.g. Coroniti 1990). In this interpretation, the orientation and eccentricity of this ring allow us to simply determine the orientation of the pulsar's spin axis in three dimensions. This information, when combined with measurements of pulsar proper motions and pulse polarization, can provide unique insight into pulsar emission mechanisms and neutron star formation (Helfand et al. 2001; Lai et al. 2001). Significant brightness variations can be seen around the perimeters of these termination shocks. If interpreted as resulting from Doppler boosting, these brightness variations yield the velocity of the post-shock flow, which in turn can be used to infer the ratio of magnetic to particle energy in the shocked wind ( $\mathrm{Lu}$ et al. 2002). In some sources, the termination shock region shows a complex morphology, comprised of multiple concentric wisps or arcs; this may represent internal shock structure, as can be produced by gyrating ions in the wind (Gaensler et al. 2002a). Chandra observations are also now beginning to reveal remarkable time-variability in these regions, demonstrating the highly dynamic nature of the relativistic flow (e.g. Hester et al. 2002). Clearly all these various compact features provide our best diagnostic yet of the nebular energetics and structure.

While results from Chandra have strikingly verified that bubble PWNe indeed have the basic structure shown in Figure 1, these observations have also demonstrated that the assumptions of isotropy and sphericity also shown in this Figure are highly questionable. Most spectacular are the bright and collimated "jets" seen for several sources as shown in Figure 1, all oriented along the symmetry axis defined by the inner toroidal termination shock. These features have a wide range in brightnesses and extents, so one should be cautious of applying a blanket interpretation to them. However, the fact that these structures have a symmetry which aligns with that seen in the termination shock region argues that they lie (at least in projection) along the pulsar spin axis. While some authors have argued that these structures are not true jets (e.g. Radhakrishnan \& Deshpande 2001), direct observations show material flowing along these structures at $v \gtrsim 0.3 c$ (Hester et al. 2002), while the lack of synchrotron cooling inferred from their spectra requires a similarly high flow speed (Gaensler et al. 2002a). In some cases these jets are distinctly one-sided; it is not yet clear whether this is due to Doppler boosting, or whether there is an intrinsic asymmetry in these outflows. It is also not clear how these jets are formed or collimated, 
but presumably the magnetic field of the neutron star plays an important role (e.g. Lyubarsky \& Eichler 2001).

\subsection{Bow-Shock Pulsar Wind Nebulae}

When a pulsar is near the edge of or outside its associated SNR, it is the ram pressure of the pulsar's motion which now serves to confine the pulsar wind. The PWN consequently has a characteristic bow-shock morphology, whose scale is set by the stand-off distance, $R_{0}$, between the pulsar and the apex of the bow shock. Pressure balance implies:

$$
\frac{\dot{E}}{\Omega r_{w}^{2} c} \approx \rho V^{2}
$$

where $\Omega$ is the solid angle through which the wind flows, $\rho$ is the ambient density, and $V$ is the pulsar's space velocity; $R_{0}$ typically differs from the termination shock radius, $r_{w}$, by a numerical constant of order 1 (Bucciantini 2002). For warm neutral regions of the interstellar medium $\left(\rho \sim 1 \times 10^{-24} \mathrm{~g} \mathrm{~cm}^{-3}\right.$ ), the corresponding angular scale is $\sim 1^{\prime \prime}$.

The overall structure of a bow shock is shown in Figure 2. Just as for a bubble PWN, the nebula can be divided up into zones corresponding to the freely flowing wind, the shocked wind, the shocked ISM and ambient undisturbed material. In this case two separate emission mechanisms are potentially observable: collisional excitation of ambient neutrals at the outer shock can generate $\mathrm{H} \alpha$ emission, while the internal shocked pulsar wind should produce X-ray/radio synchrotron emission. Until recently, there was yet to be a pulsar in which both these regions had been identified simultaneously, but new optical/X-ray data on PSR B1957+20 (Figure 2; Stappers et al. 2003) now wonderfully demonstrate both the outer optical bow shock and the inner X-ray termination shock in the same source.

The morphology, velocity structure and brightness of a bow shock in the $\mathrm{H} \alpha$ line provide powerful diagnostics of the properties of the pulsar wind and of its interaction with the ISM. Most simply, in the idealized situation of an isotropic wind interacting with a homogeneous ambient medium, the shape of a bow shock should have a simple analytic form (Wilkin 1996):

$$
R(\theta)=R_{0} / \sin \theta \sqrt{3(1-\theta / \tan \theta)},
$$

where $R$ is the distance of the bow shock from the pulsar at an angle $\theta$ from the apex. In some cases (e.g. PSR J0437-4715; Fruchter 1995), Equation (2) provides a good match to the observed morphology; however in other sources (e.g. PSR J2124-3358; Gaensler et al. 2002b), the nebula 

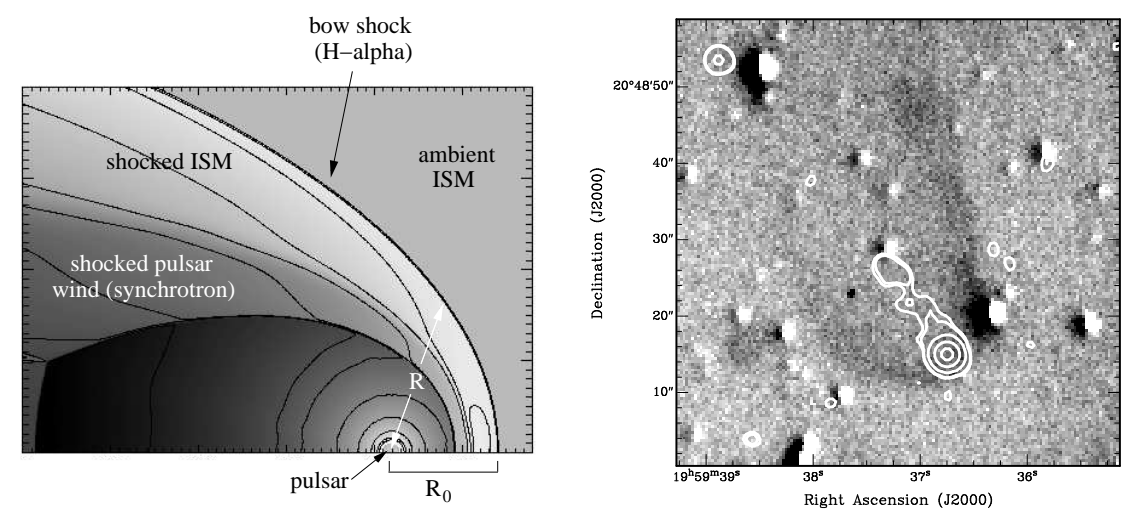

Figure 2. Cartoon of a bow-shock PWN (left; Bucciantini 2002), compared to an optical (greyscale; AAT) plus X-ray (contours; Chandra) observation of PSR B1957+20 (right; Stappers et al. 2003).

deviates strongly from this expectation, implying anisotropies in the pulsar wind and/or structure in the ISM. Additional information is starting to become available by studying the time evolution of $\mathrm{H} \alpha$ bow shocks over time-scales of $>5-10$ years. The observed changes in shape and brightness as a function of time provide a unique probe of density fluctuations in the ISM on scales of $\sim 1000 \mathrm{AU}$.

\section{Conclusions}

Interest in pulsar nebulae has resurged in the last few years, because of a dramatic increase in the quality and quantity of data across the electromagnetic spectrum. We can now be confident that we are seeing in our data the predicted shock structures for both bubble and bow-shock nebulae, and can consequently use the geometry of a PWN to infer the orientation of the pulsar's spin axis, and the magnitude and direction of the pulsar's motion. Spectral, spatial and temporal variations in the emission from PWNe now provide numerous probes of the way in which a pulsar interacts with its surroundings.

Many questions still remain unanswered. How are the prominent jets seen in several sources formed and collimated? What are the physical processes which account for the complicated compact structures and remarkable time variability now being identified in many sources? And ultimately, what are the drivers behind the diversity seen in PWN morphologies? While these are difficult questions to answer, they are now questions which we 
have the data to actually address. Clearly we have entered a new era in the study of pulsars and their winds.

\section{Acknowledgments}

I acknowledge the support of NASA through SAO grants GO2-3041X, GO23075X and GO2-3079X, and through XMM-Newton Guest Observer grant NAG5-11376.

\section{References}

1. J. M. Blondin, R. A. Chevalier, \& D. M. Frierson, ApJ, 563, 806 (2001).

2. N. Bucciantini, A\&A, 387, 1066 (2002).

3. R. A. Chevalier Mem. Soc. Astron. It., 69, 977 (1998).

4. F. V. Coroniti, ApJ, 349, 538 (1990).

5. D. A. Frail, E. B. Giacani, W. M. Goss, \& G. Dubner, ApJ, 464, L165 (1996).

6. A. S. Fruchter, http://www.stsci.edu/ fruchter/nebula/ (1995)

7. B. M. Gaensler, J. Arons, V. M. Kaspi, M. J. Pivovaroff, N. Kawai, \& K. Tamura, ApJ, 569, 878 (2002a).

8. B. M. Gaensler,D. H. Jones, \& B. W. Stappers, ApJ, 580, L137 (2002b).

9. D. J. Helfand, E. V. Gotthelf, \& J. P. Halpern, ApJ, 556, 380 (2001).

10. J. J. Hester, K. Mori, D. Burrows, J. S. Gallagher, J. R. Graham, M. Halverson, A. Kader, F. C. Michel, \& P. Scowen, ApJ, 577, L49 (2002).

11. D. Lai, D. F. Chernoff, \& J. M. Cordes, ApJ, 549, 1111 (2001).

12. F. J. Lu, Q. D. Wang, B. Aschenbach, P. Durouchoux, \& L. M. Song, ApJ, 568, L49 (2002).

13. Y. Lyubarsky, \& D. Eichler, ApJ, 562, 494 (2001).

14. R. N. Manchester \& J. H. Taylor, Pulsars, Freeman: San Francisco (1977).

15. F. C. Michel, ApJ, 158, 727 (1969).

16. J. M. Migliazzo, B. M. Gaensler, D. C. Backer, B. W. Stappers, E. van der Swaluw, \& R. G. Strom, ApJ, 567, L141 (2002).

17. F. Pacini \& M. Salvati, ApJ, 186, 249 (1973).

18. V. Radhakrishnan \& A. A. Deshpande, A\&A, 379, 551 (2001).

19. P. Slane, ASP Conf. Ser. 271: Neutron Stars in Supernova Remnants (eds Slane \& Gaensler), p165 (2002).

20. B. W. Stappers, B. M. Gaensler, V. M. Kaspi, M. van der Klis, \& W. H. G. Lewin, Science, 299, 1372 (2003).

21. M. C. Weisskopf et al. , ApJ, 536, L81 (2000).

22. F. P. Wilkin, ApJ, 459, L31 (1996).

23. L. Woltjer, M. Salvati, F. Pacini, \& R. Bandiera, A $E A$, 325, 295 (1997). 